\title{
OUR EYES CAN SEE ONLY WHAT THE MIND KNOWS: A RARE CASE OF TUBERCULOSIS OF BREAST
}

\author{
Indrani Bhattacharyya ${ }^{1}$, Jaya Das², Debopriyo Samaddar ${ }^{3}$,Sarmishtha Bandyopadhyay ${ }^{4}$ Rajyasri Guha Thakurta ${ }^{5}$
}

${ }^{1}$ Associate Professor, Department of Microbiology, CNMC, Kolkata.

${ }^{2}$ Third Year Postgraduate, Department of Microbiology, CNMC, Kolkata.

3 Demonstrator, Department of Microbiology, CNMC, Kolkata.

${ }^{4}$ Second Year Postgraduate, Department of Microbiology, CNMC, Kolkata.

5 Professor and HOD, Department of Microbiology, CNMC, Kolkata.

\section{ABSTRACT}

Tuberculous mastitis is an infrequent clinical entity. It appears mostly in women of reproductive age. We report a case of 25 year-old female presenting with complaints of cough, weight loss and intermittent episodes of fever for last 6 months. She also gave history of pain and sense of heaviness in the right breast along with discharge for last 1 month. On general physical examination, no abnormality was detected except pallor. Examination of the right breast revealed an ill-defined, irregular, firm lump (15×12 $\mathrm{cm})$, adherent to the breast tissue. Ultrasonography guided aspiration of pleural fluid and pus from breast abscess was done and sent for microbiological analysis. Acid fast bacilli were detected on microscopy of pleural fluid and aspirated pus. Mycobacterium tuberculosis was identified in both samples by using line-probe assay (GenoType MTBDRplus). RpoB gene was absent in both the samples. She gradually improved over one month of anti-tubercular treatment. This disease can present with a diagnostic dilemma on radiological and microbiological investigations and thus a high index of suspicion is essential as demonstration of Acid Fast Bacilli (AFB) from the lesions is usually difficult. Incorporating a highly sensitive technique like Polymerase Chain Reaction (PCR), Line Probe Assay (LPA) may be helpful in establishing the usefulness of such technology and can aid in confirming diagnosis early.

\section{KEYWORDS}

Mycobacterium, Acid Fast Stain, Breast Abscess, PCR.

HOW TO CITE THIS ARTICLE: Bhattacharyya I, Das J, Samaddar D, et al. Our eyes can see only what the mind knows: a rare case of tuberculosis of breast. J. Evolution Med. Dent. Sci. 2016;5(80):6009-6010, DOI: 10.14260/jemds/2016/1356

\section{INTRODUCTION}

A 25-year-old female patient presented with complaints of cough for last 6 months, weight loss and intermittent episodes of fever. She also gave history of pain and sense of heaviness in the right breast along with discharge for last 1 month. She consulted private medical practitioner for her problems and was initially put on oral Augmentin $625 \mathrm{mg}$ Q8H and Azithromycin $500 \mathrm{mg}$ Q24H for a brief period. On suspicion of pulmonary tuberculosis, she was subsequently prescribed Anti-Tubercular Treatment (ATT) even though her sputum was negative for mycobacterium tuberculosis on microscopy and GeneXpert MTB/RIF. Although, she admitted that she was on irregular ATT for last 4 months and missed several doses.

On general physical examination, no abnormality was detected except pallor. Examination of the right breast revealed an ill-defined, irregular, firm lump $(15 \times 12 \mathrm{~cm})$, adherent to the breast tissue with cystic areas involving almost the entire breast, except the upper outer part and part of the lower inner quadrant. Axillary and cervical including supraclavicular lymph nodes were not enlarged. Respiratory system examination revealed diminished breath sounds over bilateral lower zones. Sputum was negative for AFB on Z-N staining as well as on GeneXpert. Meanwhile, her chest X-ray revealed few bilateral infiltrates on lower lung.

Financial or Other, Competing Interest: None.

Submission 31-08-2016, Peer Review 24-09-2016,

Acceptance 30-09-2016, Published 06-10-2016.

Corresponding Author:

Jaya Das,

CHQ Hostel, Room No. 12,

Above Chest Building,

Chittaranjan Hospital, 24 Gorachand Road,

Kolkata-700014.

E-mail: jaya.das161@gmail.com

DOI: $10.14260 / \mathrm{jemds} / 2016 / 1356$

\section{(c) (i) $\odot$}

CT thorax was also advised, which revealed miliary nodules and bilateral pleural effusion along with right breast abscess and paravertebral abscess. Ultrasonography guided aspiration of pleural fluid and pus from breast abscess was done and sent for microbiological analysis. Acid fast bacilli were detected on microscopy of pleural fluid and aspirated pus.

Mycobacterium tuberculosis was identified in both samples by using line-probe assay (GenoType MTBDR plus). RpoB gene was absent in both the samples. Fine needle aspiration of the breast lump uncovered granulomatous mastitis, while biopsy of the lump revealed breast parenchyma with granulomas comprising of epithelioid cells, lymphocytes and Langhans giant cells, mixed with areas of haemorrhage and extensive fibrosis. There was no evidence of malignancy. These features suggested granulomatous mastitis.

Other laboratory parameters were under normal limits except haemoglobin being on lower side $(8.4 \mathrm{gm} / \mathrm{dL})$ and minor lymphocytosis 40\%. Pleural fluid Adenosine Deaminase (ADA) level was also raised (77.8 U/L). The patient was started on ATT again. She gradually improved over one month of treatment. Her symptoms subsided and improvement was noted on repeat chest X-ray. Picture $(1,2,3,4)$.

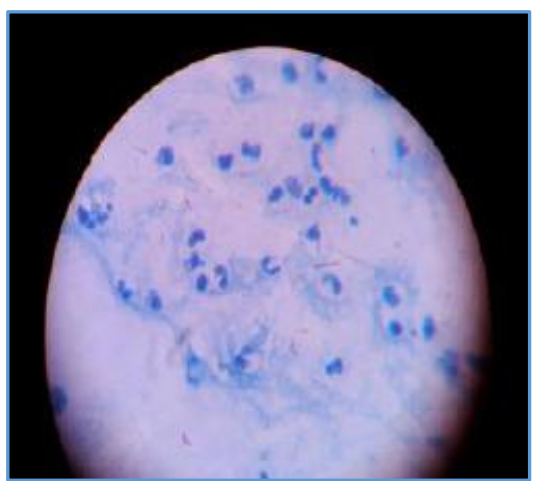

Fig. 1 


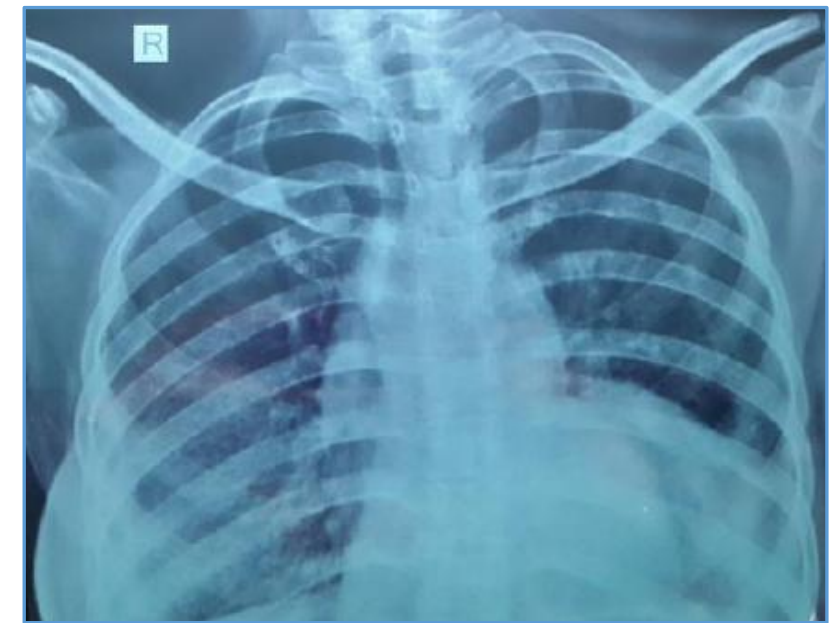

Fig. 2

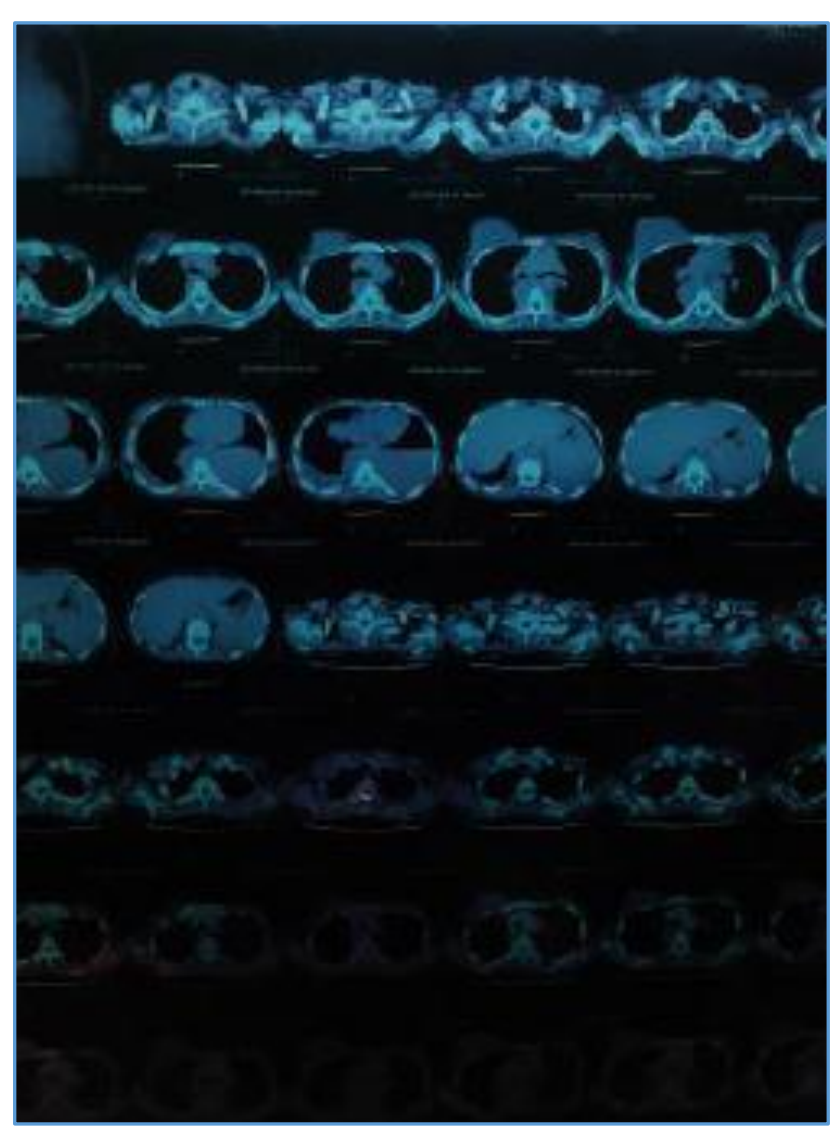

Fig. 3

\section{DISCUSSION AND CONCLUSION}

Sir Astley Cooper described the first case of mammary tuberculosis in 1829.[1] Tuberculosis of the breast is quite an uncommon occurrence and often mistaken for other lesions of the breast. The incidence of tuberculous mastitis in India has been reported to be between 1-4\%.[2,3] Tuberculous mastitis is more commonly seen in females of reproductive age group; however, especially during the lactation period when they are more susceptible since the lactating breast is more vascular and predisposed to trauma. ${ }^{[4,1]}$ Both breasts are reported to be involved with equal frequency. Bilateral disease is rare, occurring in $3 \%$ of patients.[1] The duration of symptoms varies from a few months to several years, but in most instances it is less than a year. The most common symptom is a lump in the breast. Multiple lumps are less frequent. The classical presentation with multiple sinuses, ulcers, matted nodes and a breast mass is unfortunately less common, making clinical diagnosis difficult at times. Such a presentation is seen in less than $50 \%$ of all cases. ${ }^{[5]}$ Other uncommon presentations include a typical undermined tuberculous ulcer, purulent discharge from the nipple or with a fluctuant swelling, which if inadvertently incised produces a discharging ulcer.[1,5,6]

Active or healed lung lesions on radiographic examination are rare now-a-days. Although, it was initially believed that as much as $60 \%$ of breast tuberculosis was primary, ${ }^{[5]}$ it is now accepted that mammary tuberculosis is almost invariably secondary to a lesion elsewhere in the body. Primary infection of the breast, however, through abrasions in the skin or through the duct openings on the nipple is a possibility. The most common mode of infection is thought to be retrograde lymphatic spread from the pulmonary focus through the paratracheal and internal mammary lymph nodes.[5] Coexisting tuberculosis and carcinoma of the breast was reported by Alzaraa et al.[7]

The demonstration of Acid Fast Bacilli (AFB) from the lesions is usually difficult. ${ }^{[8]}$ In tuberculous mastitis, the bacilli are isolated in only $25 \%$ of cases ${ }^{[5]}$ and AFB are identified only in $12 \%$ of the patients. Therefore, demonstration of caseating granulomas with Langhans giant cells from the breast tissue and involved lymph nodes may be sufficient for the diagnosis. In tuberculosis-endemic countries, the finding of granuloma in FNAC warrants empirical treatment for tuberculosis even in the absence of positive AFB and without culture results.[8,9] Detailed histological evaluations are however mandatory to rule out a co-existing carcinoma. Aspiration or surgical drainage may be required in some cases. In extensive cases, a simple mastectomy has been advocated. Radical mastectomy is best avoided unless there is a co-existing malignancy.[1,5,6]

\section{REFERENCES}

1. Banerjee SN, Ananthakrishnan N, Mehta RB, et al. Tuberculous mastitis: a continuing problem. World J Surg 1987;11(1):105-9.

2. Khanna R, Prasanna G, Gupta P, et al. Mammary tuberculosis: report on 52 cases. Postgrad Med J 2002;78(921):422-4.

3. Aggarwal V, Bhargava P. Breast tuberculosis-a case report. J Indian Med Assoc 2008;106(1):38-40.

4. Tewari M, Shukla HS. Breast tuberculosis: diagnosis, clinical features \& management. Indian J Med Res 2005;122(2):103-10.

5. Tauro LF, Martis JS, George C, et al. Tuberculous mastitis presenting as breast abscess. Oman Medical Journal 2011;26(1):53-5.

6. Al Soub H, Chacko K. Tuberculous mastitis: a rare disease. Br J Clin Pract 1996;50(1):50-1.

7. Alzaraa A, Dalal N. Coexistence of carcinoma and tuberculosis in one breast. World J Surg Oncol 2008;6:29.

8. Kakkar S, Kapila K, Singh MK, et al. Tuberculosis of the breast. A cytomorphologic study. Acta Cytol 2000;44(3): 292-6.

9. Mehrotra R. Fine needle aspiration diagnosis of tuberculous mastitis. Indian J Pathol Microbiol 2004;47(3):377-80. 